\title{
Prediction of Placenta Accreta Using Hyperglycosylated Human Chorionic Gonadotropin
}

\author{
Maad Mahdi Shalal ${ }^{1}$, Shaymaa Kadhim Jasim ${ }^{*}{ }^{\circledR}$, Huda Khalil Abd ${ }^{2}$
}

\begin{abstract}
Objectives: Hyperglycosylated human chorionic gonadotropin (hCG) is a variant of hCG. In addition, it has a different oligosaccharide structure compared to the regular hCG and promotes the invasion and differentiation of peripheral cytotrophoblast. This study aimed to measure hyperglycosylated hCG as a predictor in the diagnosis of placenta accreta.

Materials and Methods: In general, 90 pregnant women were involved in this case-control study among which, 30 ladies (control group) were pregnant within the gestational age of $\geq 36$ weeks with at least one previous caesarean section and a normal sited placenta in transabdominal ultrasound (TAU). The other 60 pregnant women (case group) were within a gestational age of $\geq 36$ weeks at least, one previous caesarean section and placenta previa with or without signs of placenta accreta in TAU. Hyperglycosylated hCG and total hCG were measured in each group and the results of the surgery were followed up.

Results: Hyperglycosylated hCG showed higher serum levels in patients with placenta accreta compared to those with placenta previa and control women. Hyperglycosylated hCG with an optimal cut point of (3) IU/L predicted placenta accreta in pregnant women with $90 \%$ specificity, $76.7 \%$ sensitivity, and $81.1 \%$ accuracy.

Conclusions: The high specificity of the above approach makes it a good diagnostic tool (as a single test) for confirming placenta accreta in clinical settings. When this test is added to our established workup, its high positive predictive value makes it a suitable method within the algorithm of accreta confirmation when there is a high suspicion or insufficient evidence to the diagnosis of placenta accreta.

Keywords: Hyperglycosylated human chorionic gonadotropin, Prediction, Placenta accreta
\end{abstract}

\section{Introduction}

Placenta accreta is usually used as a term with a general meaning but is defined as the level of the invasion of chorionic villi into the myometrium of pregnant ladies. Based on the depth of villous invasion, the abnormal attachment of the placenta is named placenta accreta, increta, and percreta. However, placenta previa could be defined as placenta that superimposes or is near to the internal os of the cervix $(1,2)$.

Placenta accreta may lead to massive bleeding and other morbidity issues such as acute respiratory distress syndrome, multisystem failure, intravascular coagulation (disseminated), and finally, death $(3,4)$.

Placenta accreta had an increasing incidence over the past 20-40 years, where it was about 1 per 500 in the United States, and from $0.12 \%$ during the 1970 s to $0.31 \%$ during the 2000s in Italy. It should be noted that it has an elevated frequency with cesarean sections (5-7).

The prenatal detection of placenta accreta may give a chance for multidisciplinary management before the start of any bleeding and/or labor, which is a co-factor for decreasing maternal morbidity and mortality $(7,8)$.

Some imaging methods such as transabdominal and transvaginal ultrasonography, and magnetic resonance imaging may be used to diagnose placenta accreta during pregnancy, which are limited by the need of validation evidences and the lack of generalized practicing, making them as non-accurate tools for such diagnosis, especially when the missing of a perfect serum test is added to confirm placenta accreta during the antenatal period (9-12).

Human chorionic gonadotrophin (hCG), which is the headmost circulated hormone from the placenta to the mother, appeared from about 2nd day of implantation with maximum production. The peak serum levels occur during the first trimester but remain significant throughout gestation and the low levels of hyperglycosylated human chorionic gonadotropin (hCG-H) $<50 \%$ at implantation may predict pregnancy failure $(13,14)$.

Moreover, hCG is a complex glycoprotein that consists of $\alpha$ - and $\beta$-glycosylated subunits (15). The invasive extravillous trophoblast produces hyperglycosylated forms of hCG (hCG-H), which is considered as a serum marker of an early invasion of trophoblast (16).

\section{Objective}

To foretell the diagnosis of placenta accreta by measuring hCG-H. 


\section{Materials and Methods}

A total of 90 pregnant multiparous women (18-35 years old) were involved in this case-control study and admitted to the Department of Obstetrics at Baghdad Teaching hospital in Iraq from the start of December 2017 until 31st of May 2018. This hospital is affiliated with Baghdad University. In addition, it is the grandest tertiary center in Iraq and has better resources and well-trained staff compared to the other centers. Most of the suspected cases of placenta previa and accreta from other hospitals in different states and governorates within the whole country were referred to the above hospital (tertiary center). This would explain the relatively large number of such rare cases collected during the involved period of the current study.

Their gestational ages were equal or more than 36 weeks (calculated according to the last menstrual period and the first trimester ultrasound) and the site of placenta was determined by transabdominal ultrasound (TAU). The results of the surgery were followed up to determine the number of patients ended by caesarean hysterectomy and placenta accreta was diagnosed by histopathological examination.

Patients were divided into 3 groups as follows:

- Control group: Thirty pregnant women with the normally located placenta on TAU;

- Placenta previa group: Thirty pregnant women with placenta previa but no evidence of accreta on TAU;

- Placenta accreta group: Thirty pregnant women with placenta previa and the signs of accreta on TAU.

The exclusion criteria included: 1. Pregnant women with medical diseases like hypertension in pregnancy; 2. Pregnant women with Down syndrome fetuses; 3. Pregnant women with multiple pregnancies.

Five milliliters of the entire peripheral blood was collected by venipuncture for all groups in a tube and left to clot for 30 minutes. Then, it was centrifuged (for 10 minutes) at a rate of 3000 cycles per minute, followed by collecting and freezing the serum at $-20^{\circ} \mathrm{C}$. Sera were assayed using the hCG ELISA kit (Gen Asia) and human H-HCG ELISA kit (GenAsia) according to the manufacturers' instructions.

\section{Statistical Analyses}

Discrete variables were presented using their numbers and the percentages were used to present data. In addition, the chi-square and Fisher exact tests were applied to analyze the discrete variable and the distribution between the two groups, respectively. Further, one-way ANOVA analyzed the differences between more than two groups if they followed a normal distribution with no significant outlier. Moreover, the Mann-Whitney $U$ test was performed to analyze the differences in the median of the two groups if they did not follow normal distribution while the KruskalWallis test was in the field for more than two groups. Furthermore, the linear regression analysis was carried out to assess the relationship between different variables and Person regression or Spearman correlation was implemented if one or both of them followed a normal distribution or if both of them did not follow a normal distribution, respectively. The scatter plots presented the regression analysis, as $r$ (correlation coefficient or standardized beta) is a representative of magnitude and direction of the relationship), where $r<0.25,0.25-0.5,0.5$ 0.75 , and $>0.75$ were considered as weak, mild, moderate, and assumed to have a strong correlation, respectively. A negative sign indicated an inverse relationship, but a positive sign reflected a direct relationship.

The receiver operator curve (ROC) was used to check the validity of different parameters in separating cases with accreta from the normal (control) group. The area under the curve (AUC) and its $P$ value prescribed this validity (AUC $\geq 0.9,0.8-0.89$, and 0.7-0.79 indicated excellent, good, and fair tests, respectively, and otherwise it was unacceptable). Curve calculations were done through the Trapezoidal method. The true positive rate (sensitivity) was plotted in the function of the false positive rate (100- specificity) for different cut-off points within the ROC curve. Each point on the ROC curve represented a sensitivity/specificity pair corresponding to a particular decision threshold. A test with perfect discrimination (no overlap in the two distributions) had a ROC curve that passed through the upper left corner (100\% sensitivity and $100 \%$ specificity). Therefore, the closer was the ROC curve to the upper left corner, the higher was the overall accuracy of the test. A cut-off value with a high sensitivity would be selected when the test was used to screen or exclude a diagnostic possibility while a higher specificity would be required when that test was used to confirm disease.

Finally, SPSS 20 (Statistical Package for the Social Sciences), Minitab 17.1.0, and GraphPad Prism 7.0 software were used to perform the statistical analysis and $P \leq 0.05$ was considered statistically significant.

\section{Results}

Both gestational and maternal ages for all three groups were not statistically significant. However, parity was significantly higher in both previa and accreta compared to the control group, but no significant difference was found between previa and accreta (Table 1).

Patients with accreta had higher HCG levels compared to previa and control categories although no statistical significance was observed between all 3 groups. Based on the results, patients with placenta previa and patients with placenta accreta had significantly higher levels of HCG-H in comparison to the control group, but no significant difference was detected regarding placenta previa and placenta accreta groups. The results further demonstrated that patients with accreta had a statistically significantly higher median HCG-H\% levels compared to the control group. In addition, those with previa did the same. Despite 
Table 1. Maternal Characteristics

\begin{tabular}{lccc}
\hline Variable & Control & Previa & Accreta \\
\hline Maternal age (years), mean \pm SD & $24.9 \pm 4.2$ & $24.5 \pm 5.3$ & $26.8 \pm 4.7$ \\
Gestational age (weeks), mean \pm SD & $37.5 \pm 1.2$ & $37.4 \pm 1.1$ & $37.7 \pm 1.3$ \\
Parity, median (IQR) & $1(1-2.3)$ & $2.5(1.8-4)$ & $3(2-3.3)$ \\
\hline
\end{tabular}

Note. SD: Standard deviation; IQR: Interquartile range ( $25 \%$ of the data to $75 \%$ of the data).

the fact that accreta had higher HCG-H\% levels compared to previa, there was no statistical difference between the two groups in this regard. The related data are provided in Table 2.

\section{Post Hoc Analysis}

HCG-H: Control versus previa and control versus accreta $<0.001$ and previa versus accreta 0.054 .

HCG-H\%: Control versus previa and control versus accreta $<0.001$, along with previa versus accreta 0.156 .

Women with high HCG levels were found only in the accreta group (10\%), but these results could not touch a statistical difference while there were significant differences regarding accreta results related to HCGH and HCG-H: HCG\%, the details of which are shown in Table 3.

There was a significant correlation between HCG and HCG-H only in previa and accreta groups, where it was stronger in accreta patients since the correlation coefficient was higher in this group. Conversely, the control group did not show any association between HCG and HCG-H (Table 4).

Table 5 demonstrates data on the failure of any statistically considered difference between HCG and HCG-H for all the three groups of sample patients. Nevertheless, higher relationships were documented in previa and accreta women.

By using the receiver operating characteristic curve, the AUC was 0.845 while a $95 \%$ coefficient interval was 0.753-0.912 and $P<0.001$. HCG-H could significantly discriminate between accreta and control patients at an optimal cut point of $>3$ with $90 \%$ specificity, $76.7 \%$ sensitivity, and $81.1 \%$ overall diagnostic accuracy, assuming that positive and negative predictive values were $93.9 \%$, and $65.9 \%$, respectively.

\section{Discussion}

A strictly regulated process of a normally implanted placenta should take place to have a successful pregnancy, which needs a huge number of cytokines, steroids, prostaglandins, immunologic mediators, and other factors for such successful implantation $(17,18)$. In this study, HCG-H was studied using a novel approach in order to elucidate its association with placenta accreta and previa, which were proven after cesarean delivery). The result revealed a significant elevation of HCG-H in patients with accreta compared to normal pregnancy while for the same patients' sample, the total HCG levels were not significant. Both Kovalevskaya et al (19), and Cole (20) studied the changes in the serum HCG-H and its proportion to the total HCG throughout a normal pregnancy and noted a

Table 2. Analysis of Serum HCG Levels Divided by Groups

\begin{tabular}{|c|c|c|c|c|c|}
\hline HCG Type & & Control & Previa & Accreta & $P$ Value \\
\hline \multirow{2}{*}{ HCG } & Median (IQR) & $95.0(74.8-192.5)$ & $104.2(80.0-191.0)$ & $165.0(86.0-254.7)$ & \multirow{2}{*}{0.130} \\
\hline & Range & $2.4-255.0$ & $10-221.7$ & $30.0-500.0$ & \\
\hline \multirow{2}{*}{ HCG-H } & Median (IQR) & $0.84(0.58-1.55)$ & $6.2(1.57-26.25)$ & $25.3(15.03-34.0)$ & \multirow{2}{*}{$<0.001$} \\
\hline & Range & $0.05-22.5$ & $0.05-100$ & $0.05-100$ & \\
\hline \multirow{2}{*}{ HCG-H: HCG\% } & Median (IQR) & $0.79(0.55-1.37)$ & 6.25 (1.77-22.84) & $14.95(10.0-20.0)$ & \multirow{2}{*}{$<0.001$} \\
\hline & Range & 0.11-50.0 & $0.45-61.5$ & $0.08-37.5$ & \\
\hline
\end{tabular}

Note. IQR: interquartile range; HCG: human chorionic gonadotropin; HCG-H: hyperglycosylated human chorionic gonadotropin.

Table 3. Details of Serum HCG Values for Each Group

\begin{tabular}{|c|c|c|c|c|c|}
\hline HCG Type & & $\begin{array}{l}\text { Control } \\
\text { No. (\%) }\end{array}$ & $\begin{array}{l}\text { Previa } \\
\text { No. (\%) }\end{array}$ & $\begin{array}{l}\text { Accreta } \\
\text { No. (\%) }\end{array}$ & $P$ value \\
\hline \multirow{2}{*}{ HCG } & Normal & $30(100 \%)$ & 30 (100\%) & 27 (90\%) & \multirow{2}{*}{0.0449} \\
\hline & High & $0(0)$ & $0(0)$ & $3(10)$ & \\
\hline \multirow{2}{*}{$\mathrm{HCGH}$} & Normal & 28 (93.3\%) & $17(56.7 \%)$ & $10(33.3 \%)$ & \multirow{2}{*}{$<0.001$} \\
\hline & High & $2(6.7 \%)$ & $13(43.3 \%)$ & $20(66.7 \%)$ & \\
\hline \multirow{3}{*}{ HCG-H: HCG\% } & Low & $26(86.7 \%)$ & $9(30 \%)$ & $4(13.3 \%)$ & \multirow{3}{*}{$<0.001$} \\
\hline & Normal & $1(3.3 \%)$ & $5(16.7 \%)$ & $2(6.7 \%)$ & \\
\hline & High & $3(10 \%)$ & $16(53.3 \%)$ & $24(80 \%)$ & \\
\hline
\end{tabular}

Note. HCG: human chorionic gonadotropin; HCG-H: hyperglycosylated human chorionic gonadotropin. 
Table 4. Correlation Between HCG and HCG-H

\begin{tabular}{lcc}
\hline Patients' Group & Correlation Coefficient & $\boldsymbol{P}$ Value \\
\hline Control & -0.107 & 0.573 \\
Previa & 0.565 & 0.001 \\
Accreta & 0.808 & $<0.001$ \\
\hline
\end{tabular}

Note. HCG: human chorionic gonadotropin; HCG-H: hyperglycosylated human chorionic gonadotropin.

Table 5. Correlation Between HCG and HCG-H:HCG\% Ratio

\begin{tabular}{lcc}
\hline Patients' Group & Correlation Coefficient & $\boldsymbol{P}$ Value \\
\hline Control & -0.355 & 0.055 \\
Previa & 0.274 & 0.142 \\
Accreta & 0.169 & 0.371 \\
\hline
\end{tabular}

Note. HCG: human chorionic gonadotropin; HCG-H: hyperglycosylated human chorionic gonadotropin.

rapid decline in this proportion across gestation with the following pattern.

At blastocyst implantation and the time of missed menses it was $94 \%$ and $68 \%$, respectively. After 5, 6, and 7-12 weeks of pregnancy, it was 50\%, 25\%, and $10 \%$, and during the rest of a balanced normal pregnancy, it was less than $2 \%$. They reported that this decline was caused by the differentiation of 2 to 4 nucleus syncytiotrophoblast cells into 20 to 30 nucleus cells with limited cytoplasm and endoplasmic reticulum for making proteins. In our study, $80 \%$ of accreta patients had an elevated HCG-H proportion indicating dysregulation in the natural declining process of HCG-H in pregnant women with placenta accreta, which could be attributed to the biological role of HCG-H in promoting implementation.

It is evidently known that HCG-H acts to promote the invasion of the myometrium and other tissues in patients with choriocarcinoma (a trophoblastic disease) since it is the principle HCG variant that is produced in the cells of choriocarcinoma $(21,22)$. It was theorized that the increase in the serum HCG-H might be produced by invasive placental tissues. This makes cytotrophoblast and extravillous cytotrophoblast cells to continue the secretion of HCG-H and a consequent result of cytotrophoblast cell growth, and myometrium tissue invasion or even deeper tissue infiltration in increta and percreta cases could be observed since these autocrine properties of HCG-H would promote its own production.

The findings of this study revealed that HCG-H was positively and strongly correlated with placenta accreta and previa. However, it was stronger (statistically significant) in the placenta accreta while this relationship did not exist in normal pregnant women. Accordingly, this may consolidate our conclusion of such a disproportional increase in HCG-H in accreta patients.

These observations may put this study in a unique position as the first reported article that recognized HCG-H as a diagnostic test for predicting placenta accreta.
With a cut point of the HCG-H level of more than 3 IU/L (international unit per liter), we were $90 \%$ specific, $76.7 \%$ sensitive, and $81.1 \%$ accurate to diagnose placenta accreta. Only a recently published paper (12), stated that HCG-H levels were lower in placenta accreta gravidas compared to the controls during the 2nd and 3rd trimesters of pregnancy. Related data were collected retrospectively and the researchers confirmed their findings regarding lower HCG-H levels due to weak implantation over abnormal decidua. In fact, it is well known that poor trophoblastic implantation is associated with higher rates of preeclampsia rather than placenta accreta $(23,24)$.

The high specificity of the test could pave the way to consider it as a possibly reliable single diagnostic test for confirming placenta accreta in the clinical setting. Further, considering its high positive predictive value (93.9\%) in our patients' data, the HCG-H test may be considered as a suitable tool to be added to the established algorithm of accreta confirmation when there is a high suspicion or insufficient evidence to diagnose placenta accreta, including patients with high risk (as in women with placenta previa and a previous history of cesarean delivery) or those with placental positions away from lower uterine area.

\section{Conflict of Interests}

Authors declare that they have no conflict of interests.

\section{Ethical Issues}

This study was approved by the College of Medicine Institutional Review Board, University of Baghdad.

\section{Financial Support}

None.

\section{Acknowledgments}

The authors would like to thank all medical staff and sub-staff of Medical City Hospital for their kind help and support.

\section{References}

1. Goh WA, Zalud I. Placenta accreta: diagnosis, management and the molecular biology of the morbidly adherent placenta. J Matern Fetal Neonatal Med. 2016;29(11):1795800. doi: 10.3109/14767058.2015.1064103

2. Silver RM, Fox KA, Barton JR, et al. Center of excellence for placenta accreta. Am J Obstet Gynecol. 2015; 212(5): 561-8. doi:10.1016/j.ajog.2014.11.018.

3. Belfort MA. Placenta accreta. Am J Obstet Gynecol. 2010; 203: 430-9. doi: 10.1016/j.ajog.2010.09.013

4. Wortman AC, Alexander JM. Placenta accreta, increta, and percreta. Obstet Gynecol Clin North Am. 2013;40(1):13754. doi: 10.1016/j.ogc.2012.12.002

5. Wu S, Kocherginsky M, Hibbard JU. Abnormal placentation: twenty-year analysis. Am J Obstet Gynecol. 2005;192(5):1458-61. doi: 10.1016/j.ajog.2004.12.074

6. Morlando M, Sarno L, Napolitano R, et al. Placenta accreta: 
Incidence and risk factors in an area with a particularly high rate of cesarean section. Acta Obstet Gynecol Scand. 2013;92(4): 457-60. doi:10.1111/aogs.12080.

7. Eller AG, Porter TF, Soisson P, Silver RM. Optimal management strategies for placenta accreta. BJOG. 2009;116(5):648-54. doi: 10.1111/j.1471-0528.2008.02037.x

8. Abuhamad A. Morbidly adherent placenta. Semin Perinatol. 2013;37:359-64. doi: 10.1053/j.semperi.2013.06.014

9. Committee on Obstetric Practice. Committee opinion no. 529: placenta accreta. Obstet Gynecol. 2012;120(1):207. doi:10.1097/AOG.0b013e318262e340.

10. Calì G, Giambanco L, Puccio G, Forlani F. Morbidly adherent placenta: Evaluation of ultrasound diagnostic criteria and differentiation of placenta accreta from percreta. Ultrasound Obstet Gynecol. 2013;41(4):406-12. doi:10.1002/uog.12385.

11. Shih JC, Jaraquemada JMP, Su YN, et al. Role of threedimensional power Doppler in the antenatal diagnosis of placenta accreta: Comparison with gray-scale and color Doppler techniques. Ultrasound Obstet Gynecol. 2009;33(2):193-203. doi:10.1002/uog.6284.

12. Einerson BD, Straubhar A, Soisson S, et al. Hyperglycosylated hCG and placenta accreta spectrum. 2019;36(1):22-26. doi: 10.1055/s-0038-1636501.

13. Sasaki Y, Ladner DG, Cole LA. Hyperglycosylated human chorionic gonadotropin and the source of pregnancy failures. Fertil Steril. 2008;89(6):1781-6. doi: 10.1016/j. fertnstert.2007.3.010

14. Fournier T, Guibourdenche J, Evain-Brion D. Review: hCGs: Different sources of production, different glycoforms and functions. Placenta. 2015;36:S60-5. doi:10.1016/j. placenta.2015.02.002.

15. Fiddes JC, Goodman HM. The gene encoding the common alpha subunit of the four human glycoprotein hormones. J Mol Appl Genet. 1981;1(1):3-18.
16. Guibourdenche J, Handschuh K, Tsatsaris V, et al. Hyperglycosylated hCG is a marker of early human trophoblast invasion. J Clin Endocrinol Metab. 2010;95(10): E240-4. doi:10.1210/jc.2010-0138.

17. Evans J. Hyperglycosylated hCG: a Unique Human Implantation and Invasion Factor. Am J Reprod Immunol. 2016;75(3): 333-40. doi:10.1111/aji.12459.

18. Cole LA, Khanlian SA, Sutton JM, Davies S, Stephens ND. Hyperglycosylated hCG (Invasive Trophoblast Antigen, ITA) a key antigen for early pregnancy detection. Clin Biochem. 2003;36(8):647-55. doi:10.1016/S00099120(03)00108-5.

19. Kovalevskaya G, Birken S, Kakuma T, et al. Differential expression of human chorionic gonadotropin (hCG) glycosylation isoforms in failing and continuing pregnancies: Preliminary characterization of the hyperglycosylated hCG epitope. J Endocrinol. 2002;172(3):497-506. doi:10.1677/ joe.0.1720497.

20. Cole LA. Biological functions of hCG and hCG-related molecules. Reprod Biol Endocrinol. 2010;8(1):102. doi: 10.1186/1477-7827-8-102

21. Fournier T. Human chorionic gonadotropin: Different glycoforms and biological activity depending on its source of production. Ann Endocrinol (Paris). 2016;77(2):75-8. doi:10.1016/j.ando.2016.04.012.

22. Cole LA. Hyperglycosylated hCG, a review. Placenta 2010; 31(8): 653-64. doi: 10.1016/j.placenta.2010.06.005

23. Makris A, Thornton C, Thompson J, et al. Uteroplacental ischemia results in proteinuric hypertension and elevated sFLT-1. Kidney Int. 2007;71(10):977-84. doi:10.1038/ sj.ki.5002175.

24. Phipps E, Prasanna D, Brima W, Jim B. Preeclampsia: Updates in Pathogenesis, Definitions, and Guidelines. Clin J Am Soc Nephrol. 2016;11(6):1102-13. doi: 10.2215/ cjn. 12081115

(C) 2020 The Author(s); This is an open-access article distributed under the terms of the Creative Commons Attribution License (http:// creativecommons.org/licenses/by/4.0), which permits unrestricted use, distribution, and reproduction in any medium, provided the original work is properly cited. 